\title{
XPS STUDIES ON SURFACES OF FIELD-EMISSION CATHODES OF ARTIFICIAL CARBON MATERIALS
}

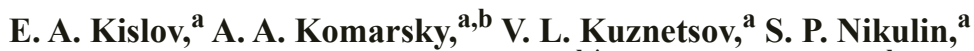 \\ D. S. Skomorokhov, ${ }^{a}$ A. S. Chepusov, ${ }^{a, b^{*}}$ and S. O. Cholakh ${ }^{b}$
}

UDC 538.971

The electronic structure of the surface layer of artificial carbon-based materials operated as field emission cathodes was studied using x-ray photoelectron spectroscopy. Samples B-1300, B-1500, and B-1900 were investigated. An analysis of the spectra showed an alteration of the hybridization of atoms in the original material, which suggested the formation of diamond-like inclusions on the sample surface.

Keywords: field-emission cathode, carbon-based materials, x-ray photoelectron spectroscopy, Auger-spectroscopy, plasmons, hybridization.

Introduction. The fabrication of stable field-emission cathodes (FECs) capable of operating for a long time under vacuum $\left(p \approx 10^{-4}-10^{-5} \mathrm{~Pa}\right.$ ) is one of the most crucial problems of modern electronics. The advantages of FECs over other types of free-electron sources include the lack of incandescence, high current density, stability to temperature fluctuations, low sensitivity to external radiation, zero lag, and exponentially high curvature of the voltammetric characteristics (VAC). These properties taken together are responsible for the promising use of FECs in various electronic instruments such as electron-beam instruments, light sources, flat screens, etc. The principal difficulty in fabricating stable FECs is the fact that field-emission of an electron is exceedingly sensitive to alternations of the cathode geometry and its surface condition.

Since the time that researchers [1] of FECs based on carbon fibers pointed out the multitude of advantages of such cathodes (long lifetime and simple preparation, potential in principle for commercial instruments), the interest in carbonbased materials $(\mathrm{CM})$ has risen significantly. The field-emission properties of a graphite cathode are determined mainly by the parameters of the microscopic projections on its emitting surface $[2,3]$. Therefore, the field-emission properties and surface structure are compared during studies of the emission properties of various types of FECs. CM have several advantages over metallic FECs used in vacuum-electron devices. These are the high melting point, good vacuum properties, cost effectiveness, availability, developed production technology, avoidance of the need to prepare an open electrode, ability to form a stable surface configuration with a significant number of emitting centers, and a stable chemical composition. CM are interesting because their structure is well known. However, the processes occurring on their surface through the action of ionizing radiation, to the effect of which the cathodes are subjected, are poorly investigated.

Residual gases are present in the voids of any electro-vacuum device. They are ionized in the space between the anode and cathode during operation through the action of electrons and bombard the cathode surface. This process can alter the cathode surface microstructure and lead to generation or destruction of emissive centers during field emission. Furthermore, the electronic structure of the cathode material can rearrange.

The processes related to cathode operation are determined by both the chemical composition of the surface and the electronic structure of the sample. The chemical composition and electronic structure can be analyzed using x-ray photoelectron spectroscopy (XPS). The present work focuses on the features of the electronic structure of the surface layer of FECs prepared from artificial CM (ACM). Samples made of coke-pitch compositions with various vitrification temperatures, B-1300, B-1500, and B-1900 [the number in the designation is the sample synthesis temperature $\left({ }^{\circ} \mathrm{C}\right)$ ], were examined. Measurements were made on an ES IFM-5 spectrometer. Two sides of the samples were studied. These were the S1 side, which operated as the field emitter, and the S2 side, which was the reverse side used as a control.

\footnotetext{
${ }^{*}$ To whom correspondence should be addressed.
}

${ }^{a}$ Institute of Electrophysics, Ural Branch, Russian Academy of Sciences, 106 Amundsen Str., Ekaterinburg, 620016, Russia; e-mail: chepusov@iep.uran.ru; ${ }^{b}$ Ural Federal University, Ekaterinburg, Russia. Translated from Zhurnal Prikladnoi Spektroskopii, Vol. 80, No. 2, pp. 216-220, March-April, 2013. Original article submitted November 1, 2012 
Experimental. The apparatus that was described before [4] was used to study the field emission properties of the $\mathrm{ACM}$. The VAC of the examined samples were obtained at pressure $\sim 10^{-4} \mathrm{~Pa}$. The long-term stability of cathode operation was studied experimentally. The long-term characteristics reflected the dynamics of the emission process under the given conditions, which were varied in the following ranges. The emission current was controlled by a power source from 0.1 to 0.3 $\mathrm{mA}$; pressure, from $2 \cdot 10^{-4}$ to $2 \cdot 10^{-2} \mathrm{~Pa}$ by admitting argon into the vacuum volume. Thus, ion bombardment of the studied cathodes was modeled. A comparison of the VAC before and after the ion irradiation demonstrated that the emission started at different potentials (the electric field potential at which the field-emission current was $25 \mu \mathrm{A}$ ). This phenomenon was probably related to rearrangements on the $\mathrm{CM}$ surface as a result of the ion bombardment.

Because the examined phenomena, i.e., field emission and modification of the sample material, occurred on the surface, the appropriate methods for studying them were required. One of the best methods for solving this problem was XPS. The XPS method is now well developed, has high resolving power, and is widely applied for revealing the chemical condition and bonding of the studied sample. The characteristic x-ray radiation of aluminum $\mathrm{Al} k_{\alpha 1,2}$ of energy $1486.6 \mathrm{eV}$ was used to excite the samples. Spectra were plotted as the intensity as a function of binding energy $E_{\mathrm{b}}$, which was calculated from the Fermi energy taken as zero $\left(E_{\mathrm{F}}=0\right)$.

Results and Discussion. A broad spectrum over a wide range of binding energies is usually recorded first during sample analysis. The chemical composition of the surface and its transformations during the studies can be judged from such spectra. Strong spectral lines of carbon and oxygen from $\mathrm{CO}_{2}$, water vapor, and hydrocarbons adsorbed to the surface layer in addition to lines of indium and nitrogen were observed in spectra of the reverse (nonworking) side. These impurities were not observed on the working side. However, oxygen was present and spectral lines of F, Ba, Pb, Ag, and $\mathrm{Na}$ appeared. These were possibly residual species from synthesis of the ACM. The impurity concentrations on the cathode operating side were less for samples with a higher synthesis temperature. The oxygen concentration also decreased. Thus, we observed the result of ion etching of the sample surface. The bombardment removed from the cathode surface adsorbed substances, contamination, impurities, etc.

The principal line of carbon $(\mathrm{C} 1 s)$ as the main element of the cathode material was studied in more detail. The positions of the peaks of spectral lines at 284.5,285, and $285 \mathrm{eV}$ were characteristic of the main allotropic modifications of carbon (graphite, 1; amorphous carbon, 2; and diamond, 3; respectively) [5]. In practice, situations are often encountered where the spectrum of the $\mathrm{C} 1 s$ line itself contains several components. The asymmetry index of the core levels is indicative of this. The corresponding indices of the $\mathrm{C}$ lines of the operating and reverse sides of the sample were determined according to the literature method [6]. Figure 1 shows the resulting C spectral lines of sample B-1900 (processing included subtracting background, determining the position of the line maximum and its asymmetry index, and deconvoluting it into components by the published method [6]). The asymmetry index of side S1 was 0.21 (asymmetric line); of reverse S2, 0.08 (practically symmetric line). Judging from these data, the reverse side contained only one component that corresponded to a graphite component with $E_{\mathrm{b}}=284.5 \mathrm{eV}$ (Fig. 1a). The spectrum of the operating side (Fig. 1b) contained two components with $E_{\mathrm{b}}=$ $284.5 \mathrm{eV}$, corresponding to graphite ( $s p^{2}$-hybridization), and $E_{\mathrm{b}}=285 \mathrm{eV}$, corresponding to diamond ( $s p^{3}$-hybridization). The alteration of the hybridization was also confirmed by a change of the characteristic spectral losses.

Such energy losses in the examined samples formed a noticeable broad maximum at a distance of $20-25 \mathrm{eV}$ from the principal peak toward the side of larger binding energies. Figure 2 shows the resulting spectra of plasmon losses for sample B-1500. Rearrangement of the sample surface was characterized by a shift of the $\mathrm{C} 1 s$ line and changes in the positions of the P1-P3 features [5]. Table 1 presents the peak positions of characteristic losses.

A comparison with the literature [5] indicated that the spectrum of the operating side was typical of the original graphite structure (peak $\mathrm{C} 1 s$ at $284.5 \mathrm{eV}$ ) with $s p^{2}$-hybridization and also acquired features characteristic of the diamond structure ( $s p^{3}$-hybridization), i.e., a C1s line at $285 \mathrm{eV}$ with the corresponding energy-loss spectrum.

The XPS spectrum also contained a distribution of Auger electrons expelled from the substance [7]. Thus, the XPS and Auger-electron spectra were produced in parallel. Auger electrons are known to be formed as the result of a three-step process with double ionization of the substance atoms. Valence-band electrons are most frequently active in the recombination stage of the excited electron and transfer of the energy difference to another. Therefore, the resulting Auger-electron spectrum is a convolution of the electron-density distributions of the involved levels and has a complicated multi-component structure. The carbon Auger line is a self-convolution of the valence band and reflects its electronic structure [8]. Because these lines are the convolution of the valence band and core lines and appear on the background of a strong loss spectrum, it is more convenient to use derivatives in order to identify their features. Figure 3 shows differentiated Auger lines for sample B-1500 in coordinates of kinetic energy. 

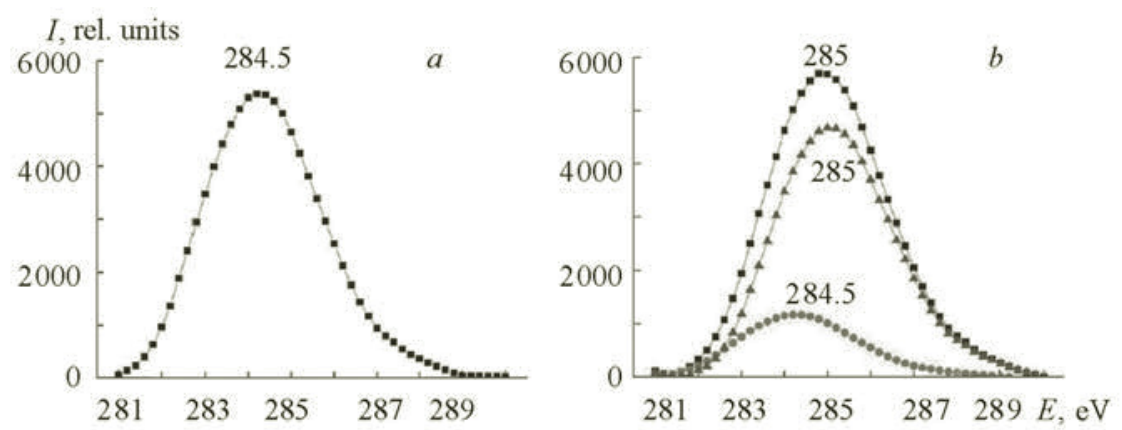

Fig. 1. C1s spectrum of sample B-1900: reverse side (S2) (a); working side (S1) (b) with deconvolution into components $284.5(\bullet)$ and $285.0 \mathrm{eV}(\boldsymbol{\Delta})$.
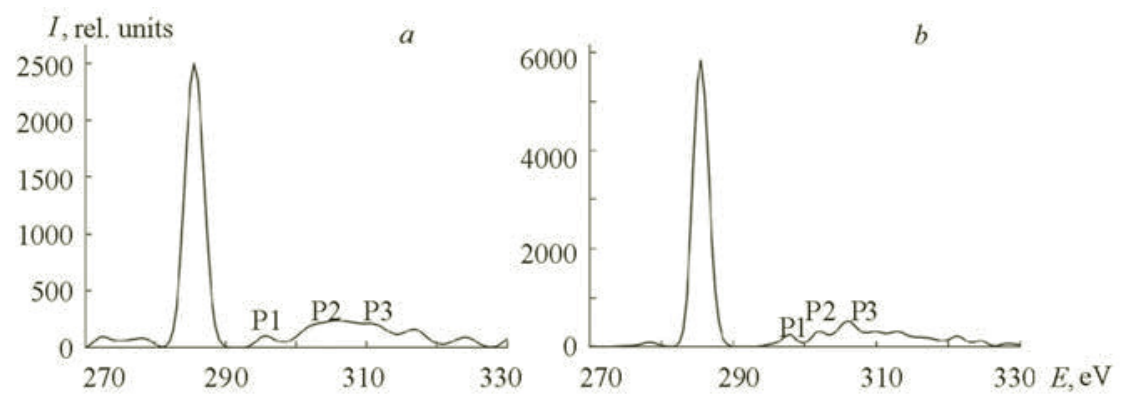

Fig. 2. Spectrum of characteristic energy losses of sample B-1500: for reverse side (S2) (a); for working side (S1) (b).

TABLE 1. Positions (eV) of Plasmon Peaks of Studied Samples

\begin{tabular}{|c|c|c|c|c|}
\hline \multirow{2}{*}{ Side } & \multirow{2}{*}{$\mathrm{C} 1 s$ position } & \multicolumn{3}{|c|}{ Distance of plasmons, $\mathrm{eV}$} \\
\hline & & $\mathrm{P} 1$ & $\mathrm{P} 2$ & P3 \\
\hline \multicolumn{5}{|c|}{ Sample B-1300 } \\
\hline Working, S1 & 285.1 & 9 & 14.5 & 22.5 \\
\hline Reverse, S2 & 284.5 & 5.5 & 20 & 23 \\
\hline \multicolumn{5}{|c|}{ Sample B-1500 } \\
\hline Working, S1 & 284.9 & 11.5 & 16.5 & 21 \\
\hline Reverse, S2 & 284.5 & 10 & 20 & 25.5 \\
\hline \multicolumn{5}{|c|}{ Sample B-1900 } \\
\hline Working, S1 & 284.9 & 9 & 19 & 26.5 \\
\hline Reverse, S2 & 284.6 & 11.5 & 20.5 & 32 \\
\hline
\end{tabular}

The shape of a positive peak of the Auger-line derivative determined the density of states in the carbon valence band. The position of a negative peak indicated the presence of a band gap. The difference in the position of a negative Auger-line peak allowed a change in the hybridization type to be found during field emission [8]. The shifts of the Auger lines for samples B-1300, B-1500, and B-1900 were 0.5, 1.5, and $2.5 \mathrm{eV}$, respectively. A study of the Auger-electron spectra demonstrated the formation of a band gap in the surface layer of the carbon FEC through the action of ion bombardment as the synthesis temperature increased. 


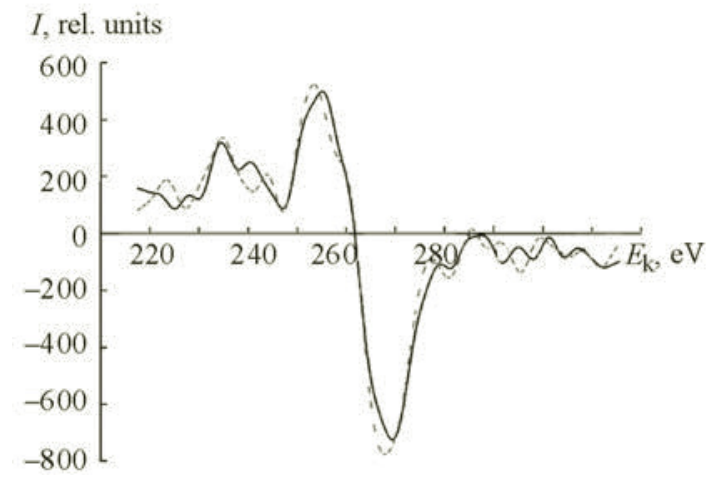

Fig. 3. Differentiated Auger-lines of sample B-1500: working side (S1) (solid line); reverse side (S2) (dashed line).
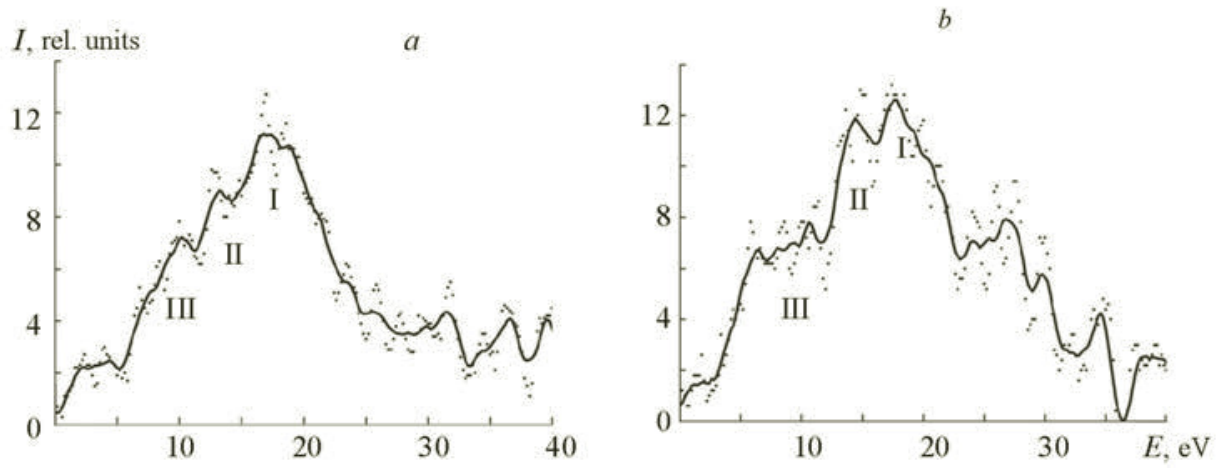

Fig. 4. Valence band of sample B-1900: reverse side (S2) (a); working side (S1) (b) after exposure to argon ions at pressure $2 \cdot 10^{-3} \mathrm{~Pa}$; lines are the running averages.

The next task was to study the valence band of these samples. The valence bands of diamond and amorphous and crystalline carbon were examined before by XPS [5]. A review of the published spectra showed that all three samples gave the same overall structure. Each spectrum had a rather broad and strong peak between 16 and $21 \mathrm{eV}$ (peak I), a narrower and weaker peak from 10 to $15 \mathrm{eV}$ (peak II), and a broad and indistinct structure stretching from 10 to $13 \mathrm{eV}$ and to the Fermi level (peak III). However, there were slight and important differences in the spectra. Peak I for diamond was less distinct than its analog for amorphous and crystalline carbon. Furthermore, peak III was sharply defined in the diamond spectrum whereas it decayed slowly as a tail toward lower energies in the graphite spectrum. Also, there was a clearly defined minimum between peaks I and II in the graphite spectrum. This persisted even for diamond. The researchers indicated that these differences were due to both the density of states and effects of the field-emission cross-section [5].

Figure 4 shows the valence bands of the operating and reverse sides of sample B-1900 after exposure to ions at working pressure $2 \cdot 10^{-3} \mathrm{~Pa}$. Line I on the reverse side had a maximum at $17 \mathrm{eV}$, was rather broad, and was stronger than peak II $(12.5 \mathrm{eV})$. A small maximum in the range of peak III decayed slowly toward lower frequencies. Such a configuration of the valence band corresponded to the crystalline carbon structure. Maxima of peaks I $(18 \mathrm{eV})$ and II $(15 \mathrm{eV})$ in the spectrum of the operating side became more distinct and differed little in intensity. The region of peak III was clearly formed. All these factors confirmed that a diamond-like structure had formed on the sample surface. The electronic structure of the ACM valence band was altered as a result of ion bombardment of the cathode during operation. This led to the formation of diamond-like clusters. The formation of such defects explained the reduction in the start of emission of the carbon cathodes.

Conclusion. Samples of ACM operating as FECs were studied by XPS. An analysis of the results led to the conclusion that inclusions with $s p^{3}$-hybridization had formed on the surface of the carbon-based material. This was confirmed by spectral features such as the changes of the $\mathrm{C} 1 s$ line position and asymmetry, the presence of several components, a change of the plasmon-loss spectrum, the formation of band gaps, and, finally, the valence-band features. 
Acknoledgments. We are deeply grateful to Prof. L. A. Pesin for supplying the samples; I. V. Gribov, Institute of the Physics of Metals, Ural Branch, RAS, for performing the spectrometry; students of UFU for participating in data processing; and the staff of LIII, IEF, UB, RAS, for help and support. The work was supported by the Russian Foundation for Basic Research, Grant No. 10-08-00830-a.

\section{REFERENCES}

1. F. S. Baker, A. R. Osborn, and J. Williams, Nature, 239, 96-97 (1972).

2 B. V. Bondarenko, V. I. Makukha, and E. P. Sheshin, Elektron. Tekh., Ser. 1, Elektronika SVCh, No. 10, $44-47$ (1984).

3. A. L. Suvorov, E. P. Sheshin, and V. V. Protasenko, Zh. Tekh. Fiz., 66, No. 7, 156-160 (1996).

4. E. A. Kislov, V. L. Kuznetsov, D. S. Skomorokhov, V. A. Terent'eva, and A. L. Filatov, Al'tern. Energ. Ekol., No. 3, 40-43 (2010).

5. F. R. McFeely, S. P. Kowalczyk, L. Ley, R. G. Carell, R. A. Pollak, and D. A. Shirley, Phys. Rev. B: Solid State, 9, No. 12, 5268-5278 (1974).

6. Yu. A. Babanov, I. Yu. Kamenskii, V. L. Kuznetsov, S. S. Mikhailova, P. V. Titov, and A. L. Filatov, Poverkhnost, No. 11, 44-48 (2006).

7. D. P. Woodruff, T. A. Delchar, and J. E. Rowe, Phys. Today, 41, No. 3, 90 (1988).

8. A. A. Galuska, H. H. Madden, and R. E. Allred, Surf. Sci., 32, No. 3, 253-272 (1988). 